\title{
Korrekturosteotomien am distalen Radius
}

\author{
Karl-Josef Prommersberger, Ulrich Lanz
}

\section{Zusammenfassung}

Trotz aller Fortschritte in der Behandlung der distalen Radiusfraktur ist die Ausheilung in Fehlstellung weiterhin eine häufige Komplikation des körperfernen Speichenbruchs. Die meisten wenn auch nicht alle - Patienten mit einer fehlverheilten distalen Radiusfraktur klagen über Bewegungseinschränkungen, Schmerzen und eine Kraftminderung ihres Handgelenkes. Ziel operativer Eingriffe bei fehlverheilten distalen Radiusfrakturen sind die Funktionsverbesserung und Schmerzreduktion. Bei der Entscheidung, ob eine fehlverheilte Fraktur des distalen Radius operativ behandelt werden soll, ist in erster Linie der Patient mit seinen funktionellen Bedürfnissen und seiner Motivation zu berücksichtigen. Ausschlaggebend bei der Frage, wie gegebenenfalls operativ vorgegangen werden kann, sind eher lokale Faktoren. Form und Länge des Radius - als Voraussetzung für eine möglichst normale Funktion können nur durch eine Korrekturosteotomie des Radius unter Interposi-

\section{Einleitung}

Trotz aller Fortschritte in der Frakturbehandlung ist die Ausheilung in Fehlstellung weiterhin eine der häufigsten Komplikationen distaler Radiusfrakturen. Hingegen ist die ausbleibende knöcherne Konsolidierung, also die Falschgelenkbildung, nach einem körperfernen Speichenbruch extrem selten. Sowohl nach extra- als auch nach intraartikulären

OP-JOURNAL 2003; 19: 58-64

(C) Georg Thieme Verlag Stuttgart - New York tion eines Knochenblockes wiederhergestellt werden. Trotz aller Unterschiede bei der Durchführung ( $\mathrm{Zu}$ gang, Osteosynthesematerial, Auffüllen des Osteotomiespaltes, etc) sind die vorliegenden Ergebnisse nach Radiuskorrekturosteotomien allgemein gut: die Schmerzen nehmen ab, es kommt zu einer Steigerung der groben Kraft und einer Verbesserung der Beweglichkeit des Handgelenkes sowie der Unterarmdrehung. Die Veränderungen der klinischen Situation von prä- nach postoperativ sind einerseits statistisch signifikant und andererseits abhängig vom radiologischen Ergebnis. Aufgrund der allgemein guten Ergebnisse muss man heute Patienten mit einer fehlverheilten distalen Radiusfraktur bei entsprechenden Beschwerden eine Radiuskorrekturosteotomie anbieten. Ziel des Eingriffs muss die möglichst anatomiegerechte Wiederherstellung des Radius in Form und Länge sein, denn die klinischen Ergebnisse sind umso besser, je günstiger das radiologische Ergebnis ausfällt.

\section{Art der Fehlstellung}

Heilt eine extraartikuläre Fraktur der körperfernen Speiche in Fehlstellung aus, so findet sich in den meisten Fällen eine mehrdimensionale Deformität mit

- Verkippung der Radiusgelenkfläche in der Sagittalebene mit Neigung nach dorsal (fehlverheilte Colles-Frakturen) (Abb.1) oder vermehrter Kippung zur Beugeseite (fehlverheilte Smith-Frakturen)

- Abflachung der Ulnarinklination in der Frontalebene

- und Verkürzung der Speiche gegenüber der Elle.

Nach eigenen Untersuchungen besteht zusätzlich in $50 \%$ der fehlverheilten Colles-Frakturen und in 75\% der fehlverheilten Smith-Frakturen ein Rotationsfehler des körperfernen Speichenfragmentes in Relation zum Radiusschaft. Die meisten Rotationsfehler sind jedoch von geringem Ausmaß und haben entsprechend funktionell wenig Bedeutung. Auch Verschiebungen des distalen Fragmentes nach dorsal bzw. palmar oder/und nach radial werden beobachtet. Häufigkeitsangaben von $50 \%$ und mehr können wir jedoch nicht bestätigen.

Intraartikuläre Frakturen des distalen Radius können mit Gelenkstufen oder/und Spaltbildungen der Gelenkfläche ausheilen. Diese können das Radiokarpal- und/ oder das distale Radioulnargelenk betreffen. Meist findet sich neben der intraauch eine extraartikuläre Fehlstellungskomponente.

und Gipsruhigstellung behandelt wurden. Ursache ist weniger eine primär ungenügende Reposition, als vielmehr eine schleichende Redislokation. Aber auch nach operativer Behandlung, selbst nach offener Reposition und interner Stabilisation, wird eine Heilung in Fehlstellung - sei es aufgrund eines sekundären Repositionsverlustes oder einer primären Fixation in Fehlstellung - beobachtet.

Speichenbrüchen kann es zur Ausheilung in Fehlstellung kommen. In der überwiegenden Mehrheit handelt es sich um Brü-

Beschwerden

Es ist zweifelsohne wahr, dass nicht alle Patienten mit einer fehlverheilten distalen Radiusfraktur Beschwerden äußern. Andererseits muss man jedoch auch feststellen, dass Patienten allgemein immer weniger bereit sind, verbliebene Funktionsbeeinträchtigungen zu tolerieren. Patienten mit einem in Fehlstellung ver- 

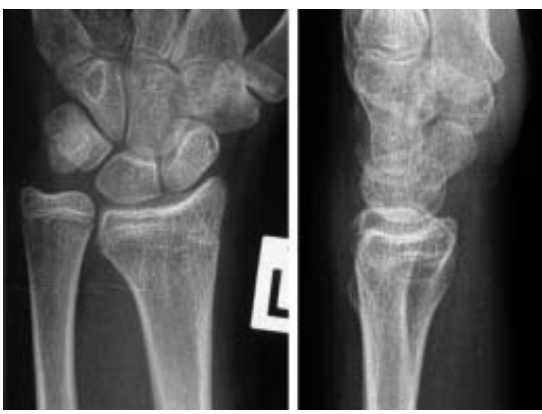

Abb.1a Ausheilung einer distalen Radiusfraktur vom Colles-Typ in typischer Fehlstellung mit Verkürzung des Radius, Abflachung der Ulnarinklination und Dorsalkippung der Radiusgelenkfläche bei einer 15-jährigen Patientin. Die Epiphysenfuge des distalen Radius ist nur noch partiell offen.

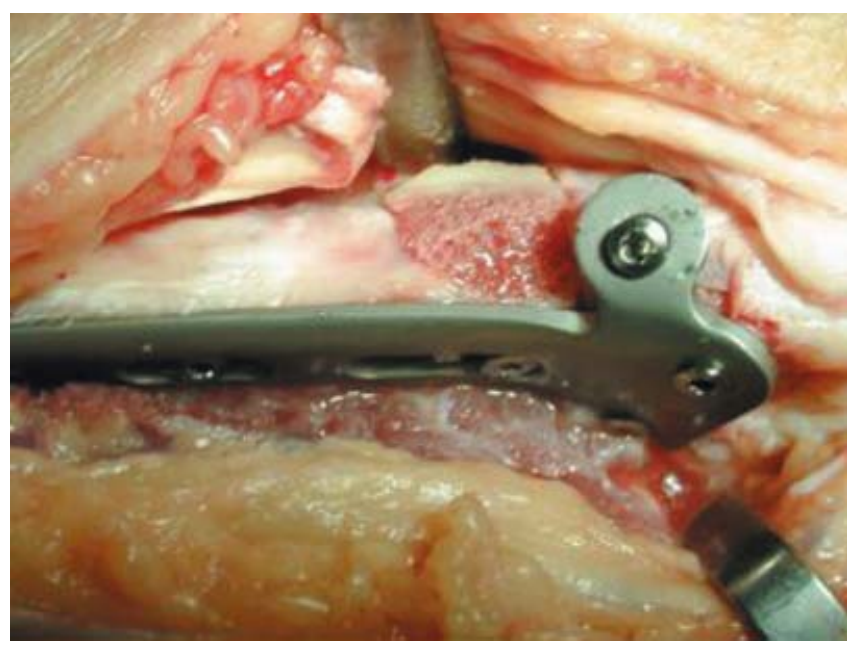

Abb.1c Radiopalmarer Zugang. Interposition eines kortikospongiösen Knochenblockes vom Beckenkamm. Stabilisation des Radius mit einer Spezialplatte für Korrekturosteotomien fehlverheilter Extensionsbrüche mit der Möglichkeit zur winkelstabilen Verschraubung distal.
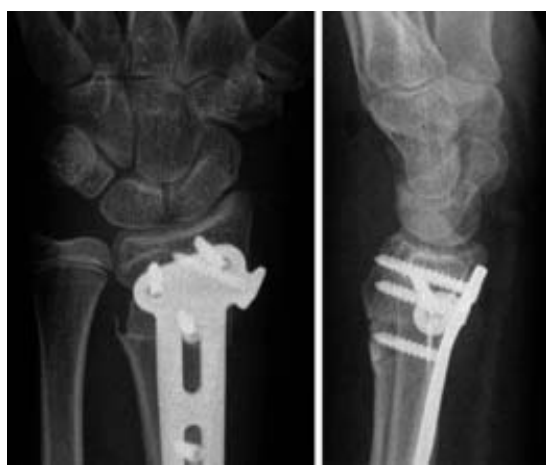

Abb.1d Frühpostoperativer Röntgenbefund: der interponierte Knochenblock ist gut sichtbar; der Radius in Form und Länge wiederhergestellt.

heilten Bruch der körperfernen Speiche klagen über

- Bewegungseinschränkungen

- Kraftminderung

- Schmerzen

- gestörte Ästhetik des Handgelenks

- Gefühlsstörungen.

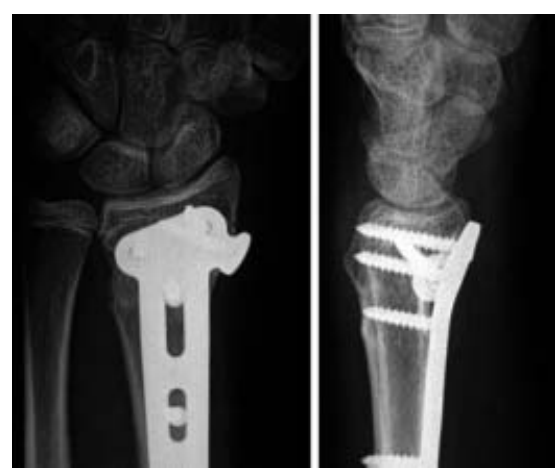

Abb.1e Vier Monate postoperativ ist der Knochenblock vollständig eingeheilt.

Die Einschränkung der Unterarmdrehbewegung, insbesondere der Supination, wird dabei als gravierender empfunden als die Einschränkung der Extension und Flexion des Handgelenkes. Der Hauptschmerzpunkt liegt - für die Patienten häufig verwirrend, jedoch durch die relative Überlänge der Ulna und das dadurch verursachte Ulna-impactionSyndrom leicht erklärbar - ulnokarpal. Das Aussehen des Handgelenkes wird und dies nicht nur von Patientinnen oft als „entstellt“ empfunden. $10 \%$ der Patienten mit fehlverheilten Speichenbrüchen berichten über Gefühlsstörungen, vorwiegend Daumen, Zeige- und Mittelfinger sowie die radiale Hälfte des Ringfingers, also das Versorgungsgebiet des Mittelnervs (N. medianus) betreffend. Subtile elektrophysiologische Untersuchungen zeigen sogar in bis zu 50\% der Fälle Beeinträchtigungen des N. medianus.

\section{Biomechanik}

Die geklagten Beschwerden der Patienten sind vor dem Hintergrund der durch die Fehlstellung des distalen Radius bedingten Störungen der Biomechanik des Handgelenkes verständlich. Diese betreffen das

- Radiokarpalgelenk

- Mediokarpalgelenk

- distale Radiokarpalgelenk (DRUG).

Bei Dorsalkippung des distalen Radiusfragmentes kommt es auf der Ebene des Radiokarpalgelenks zu einer Verlagerung der Belastungszone am Radius nach dorsal einhergehend mit einer Verschmälerung der Belastungszone. Gleichzeitig verlagert sich parallel zur Dorsalkippung des distalen Radius der Kraftfluss von der Hand auf den Unterarm hin zum ulnokarpalen Gelenkkompartiment. Erfolgt die Kraftübertragung am unverletzten, gesunden Handgelenk zu 15-30\% über die Ulna und zu 70-85\% über den Radius, so werden bei einer Dorsalkippung des distalen Radius von 30 Grad $50 \%$ der Kraft über die Ulna übertragen [10].

Auf der Ebene des Mediokarpalgelenks wird die Rotation des Lunatums, das dem distalen Radius meist in die Fehlstellung folgt, durch eine gegenläufige Kippung des Kapitatums ausgeglichen. Bei länger bestehenden Fehlstellungen verursacht dies nicht selten eine synovialitische Reizung mit entsprechenden Beschwerden. In der Regel verschwinden diese Beschwerden nach einer Korrekturosteotomie des distalen Radius. Ist die Fehlstellung der Handwurzel jedoch bereits fixiert, ist von einer Radiuskorrekturosteotomie eher abzuraten, da die karpale Fehlstellung und die dadurch verursachten Beschwerden auch nach der Korrektur der Speiche fortbestehen. 
Sowohl durch die unphysiologische Kippung des distalen Radiusfragmentes als auch durch die Verkürzung des Radius kommt es zu einer Verringerung der Kontaktfläche des distalen Radioulnargelenkes und gleichzeitig zu einem Spannungsanstieg des ulnokarpalen Komplexes. Beides kann die Unterarmdrehung beeinträchtigen.

\section{Behandlungsmöglichkeiten}

Bei der Entscheidung, ob eine fehlverheilte Fraktur des distalen Radius operativ behandelt werden soll, steht der Patient mit seinen funktionellen Bedürfnissen und seiner Motivation im Vordergrund. Hingegen sind bei der Frage, wie gegebenenfalls operativ vorgegangen werden kann, eher lokale Faktoren (Art der Fehlstellung, Knochenqualität, Arthrose, Weichteilsituation, etc) ausschlaggebend. Ziel operativer Eingriffe bei fehlverheilten distalen Radiusfrakturen ist die Schmerzreduktion und sofern möglich Funktionsverbesserung. Die Radiuskorrekturosteotomie ist lediglich eine von mehreren Möglichkeiten dieses Zieles zu erreichen.

Prinzipiell können vier Therapiegruppen unterschieden werden:

1. Eingriffe zur Wiederherstellung der normalen Anatomie

2. Eingriffe zur Funktionsverbesserung ohne Wiederherstellung der normalen Anatomie

3. Eingriffe zur Schmerzreduktion

4. Kombinationseingriffe.

Zur Reduktion von Handgelenksschmerzen nach distaler Radiusfraktur ohne wesentliche Fehlstellung hat sich die Handgelenksdenervation bewährt. Als Alternative zur vollständigen Handgelenksversteifung ist bei schmerzhafter, isolierter Radiokarpalarthrose, also bei intaktem Mediokarpalgelenk, die Fusion von Radius, Skaphoid und Lunatum zu diskutieren [1].

Von den verschiedenen Möglichkeiten zur Verbesserung der Unterarmdrehbewegung ohne Wiederherstellung anatomischer Verhältnisse hat sich in unseren Händen bei fehlverheilten distalen Radiusfrakturen die Ulnakopfhemiresektion nach Bowers [2] bewährt.

Während bei einer isolierten Verkürzung des Radius das Längenverhältnis zwischen Radius und Ulna durch eine Ulnaverkürzungsosteotomie wieder normalisiert werden kann, bedarf es bei der in der Regel vorliegenden mehrdimensionalen Fehlstellung des distalen Radius einer Korrekturosteotomie am Radius selbst, um weitgehend anatomiegerechte Verhältnisse zwischen Radius und Handwurzel, aber auch zwischen Radius und Ulna zu schaffen.

Bei Patienten mit fehlverheilten Frakturen des distalen Radius ohne Radiokarpalarthrose, aber mit Arthrose des DRUG, berichtete Fernandez [6] bereits in der 80er-Jahren über gute Ergebnisse bei Kombination einer Radiuskorrekturosteotomie mit einer Ulnakopfhemiresektion nach Bowers [2]. Heute tendiert Fernandez eher dazu, parallel zur Radiuskorrekturosteotomie eine Ulnakopfprothese in solchen Fällen einzubauen [13].

\section{Radiuskorrekturosteotomie}

\section{Indikation und Kontraindikation}

Ist aufgrund der Beschwerdesymptomatik die Indikation für ein operatives Vorgehen gegeben, so ist zu prüfen, ob eine Korrekturosteotomie des Radius im konkreten Fall das geeignete Mittel ist. Absolute radiologische Grenzwerte für eine Radiuskorrekturosteotomie existieren nicht. Die ursprüngliche Form und Länge des Radius lässt sich jedoch, wenn überhaupt, nur durch eine Osteotomie am Radius wiederherstellen. Entsprechend ist bei allen mehrdimensionalen Fehlstellungen des distalen Radius eine Radiuskorrekturosteotomie in Erwägung zu ziehen. Auch wenn bei „geringer“ Kippung der Radiusgelenkfläche von zehn Grad nach dorsal oder von 20 Grad nach palmar der hierdurch verursachte relative Ulnavorschub radiologisch im Vordergrund zu stehen scheint, sollte man nach unserer Erfahrung am Radius korrigierend eingreifen, da eine Ulnaverkürzungsosteotomie nicht die Verkantung im DRUG, sondern lediglich die Längendiskrepanz zwischen Radius und Ulna beseitigen kann.

Immer wieder wird die Frage aufgeworfen, welcher relative Ulnavorschub sich noch durch einen Eingriff am Radius allein ausgleichen lässt. Fernandez [5] berichtet, dass ab einer Radiusverkürzung von $12 \mathrm{~mm}$ eine zusätzliche Ulnaverkürzungsosteotomie erforderlich sei. Limitierender Faktor beim Längenausgleich zwischen Radius und Ulna ist der Zustand der Weichteile. Liegen keine Vernarbungen vor, lassen sich auch Radiusverkürzungen über $12 \mathrm{~mm}$ allein durch eine Radiusosteotomie unter Interposition eines Knochenblockes, gegebenenfalls nach intraoperativer Distraktion, vollständig ausgleichen. Bei kontrakten Weichteilen hingegen kann sich auch bei einem geringeren Längenverlust des Radius selbst nach Ablösung des $M$. brachioradialis der Längenausgleich problematisch gestalten.

Ein reduzierter Allgemeinzustand, eine deutliche Radiokarpalarthrose und eine massive Osteoporose sind absolute Kontraindikationen für eine Radiuskorrekturosteotomie.

Auch bei einer bereits fixierten Fehlstellung der Handwurzel infolge der fehlverheilten Radiusfraktur (s.o.), ist von einer Korrekturosteotomie am Radius abzuraten, da die Beschwerden postoperativ eher persistieren. Zeigt sich die Fingerfunktion eingeschränkt, sollte man die Radiuskorrekturosteotomie zurückstellen und primär die Fingerfunktion durch eine entsprechende krankengymnastische Übungsbehandlung verbessern. Eine abgelaufene sympathische Reflexdystrophie ohne fortbestehende trophische Störungen und ohne Einschränkungen der Fingerbeweglichkeit, ist keine Kontraindikation für eine Radiuskorrekturosteotomie, allerdings bedürfen diese Patienten postoperativ einer erhöhten Aufmerksamkeit. Auch eine leichte Instabilität des DRUG stellt keine Kontraindikation dar. Erfahrungsgemäß kommt es durch die Radiuskorrekturosteotomie zu einer Stabilisierung des DRUG [9]. Liegt eine ausgeprägte Instabilität des DRUG vor, empfiehlt sich gleichzeitig zur Radiuskorrekturosteotomie ein stabilisierender Eingriff am DRUG. Ebenso sollte man bei einer ausgeprägten Arthrose des DRUG in gleicher Sitzung neben der Radiuskorrekturosteotomie einen Eingriff am Ellenkopf, sei es eine Ulnakopfhemiresektion oder die Implantation einer Ulnakopfprothese, vornehmen.

In den 70er- und 80er-Jahren wurde die Indikation zur Radiuskorrekturosteotomie auf manuell tätige Patienten mit einem Alter unter 45 Jahren begrenzt. Angesichts der allgemein guten Ergebnisse nach Radiuskorrekturosteotomien (s.u.) wurden diese Einschränkungen fallen gelassen. In einer prospektiven Untersuchung haben wir die Ergebnisse nach Radiuskorrekturosteotomie von $16 \mathrm{~Pa}-$ tienten unter 50 Jahren (15 bis 46 Jahre, Durchschnittsalter 33) mit den Ergebnissen von 16 Patienten über 50 Jahren (51 bis 76 Jahre, Durchschnittsalter 58 ) verglichen. Die Gruppe der älteren Patienten wies einen deutlich höheren Frauenanteil auf und vermutlich hierdurch bedingt, zeigte sich die grobe Handkraft präoperativ signifikant niedriger als in der Gruppe 
der jüngeren Patienten. Ansonsten fanden sich präoperativ keinerlei Unterschiede zwischen beiden Gruppen. Durch die Radiuskorrekturosteotomie kam es in beiden Gruppen zu einer signifikanten Verbesserung aller klinischen Parameter einschließlich der Grobkraft. Trotzdem blieb die Grobkraft der älteren Patienten im Vergleich mit den jüngeren Patienten signifikant niedriger. Alle anderen klinischen und radiologischen Parameter zeigten sich auch postoperativ nahezu identisch.

Am wachsenden Skelett mit unverletzter Wachstumsfuge des distalen Radius ist die Indikation zu einer Radiuskorrekturosteotomie in Abhängigkeit von der vorhandenen Potenz zur spontanen Wachstumskorrektur einerseits und der bestehenden Funktionseinschränkung andererseits zu diskutieren. Bei partiellem oder vollständigem Epiphysenverschluss sieht man sich aufgrund der zunehmenden Deformität in der Regel gezwungen, korrigierend einzugreifen.

\section{Zeitpunkt für die Radiuskorrekturosteotomie}

Bei entsprechenden Beschwerden sollte man die Radiuskorrekturosteotomie durchführen, sobald die Schwellung abgeklungen ist und die Finger frei beweglich sind.

Beim Vergleich von Patienten, bei denen die Korrekturosteotomie im Mittel acht Wochen nach dem Unfall durchgeführt wurde, mit Patienten, bei denen der Eingriff im Schnitt erst 40 Wochen nach dem Trauma erfolgte, fanden Jupiter und Ring [7] keine Unterschiede hinsichtlich der klinischen und radiologischen Ergebnisse zwischen beiden Gruppen. Die Arbeitsunfähigkeit war jedoch in der Gruppe der frühzeitig durchgeführten Korrekturosteotomien signifikant kürzer.

\section{Operationsvorbereitung}

Die Operationsplanung erfolgt anhand von Röntgenaufnahmen beider Handgelenke in zwei Ebenen. Bei Verdacht auf einen zusätzlichen Rotationsfehler sollte - sofern ein dorsaler Zugang zum Radius vorgesehen ist - eine Computertomographie im Seitenvergleich zur Bestimmung des Rotationsfehlers durchgeführt werden. Bestehen Bedenken hinsichtlich des Knorpelzustandes oder gibt es Anhaltspunkte auf eine begleitende karpale Bandverletzung, empfiehlt sich eine Handgelenksspiegelung. Eine zeichneri- sche Planung der Osteotomie (Niveau der Osteotomie, Osteotomie- und Korrekturwinkel) ist obligatorisch.

\section{Zugang}

Korrekturosteotomien fehlverheilter Smith-Frakturen werden von allen Autoren von palmar durchgeführt (Abb.2). Bei fehlverheilten Colles-Frakturen kommen Zugänge von dorsal, radial, radiopalmar und palmar zur Anwendung.

Bereits 1937 beschrieb Campbell [3] einen radialen Zugang. Mitte der 70erJahre entwickelte Lanz [8] eine Technik zur Korrektur fehlverheilter Extensionsfrakturen unter Verwendung eines radiopalmaren Zuganges und einer Spezialplatte. Seit Einführung winkelstabiler palmarer Plattensysteme für den distalen Radius findet der rein palmare Zugang auch für die Korrekturosteotomie fehlverheilter Colles-Frakturen zunehmend Verbreitung [15]. Meistens wird jedoch der Radius immer noch wie von Fernandez $[5,6]$ beschrieben von dorsal freigelegt.

\section{Fixation}

Alle Osteosyntheseverfahren zur Stabilisation frischer Radiusfrakturen kommen auch bei Radiuskorrekturosteotomien zur Anwendung.

Der Fixateur externe kann sowohl gelenküberbrückend als auch radio-radial montiert werden, kommt jedoch deutlich seltener zum Einsatz als interne Osteosynthesen. Muss bei der Osteotomie die gegenseitige Kortikalis des Radius nicht durchtrennt werden, lässt sich bereits durch zwei Kirschner-Drähte eine ausreichende Stabilität erzielen, sofern der Osteotomiespalt mit einem gut passend zurecht geschnittenen kortikospongiösen Knochenblock aufgefüllt wird. Meistens erfolgt die Stabilisation des Radius jedoch mit einer Platte. Insbesondere bei lange bestehenden Fehlstellungen und bei einem ausgeprägten Längenverlust des Radius muss die verwendete Platte eine ausreichende Eigenstabilität aufweisen, um die auftretenden Kräfte zu neutralisieren. Ansonsten kann es zu Korrekturverlusten oder zum Bruch der Platte kommen.

\section{Was tun mit dem Osteotomiespalt?}

Durch ein langsames Aufdehnen des Radius nach der Osteotomie mittels eines Fixateur externe über Wochen kommt es zu einer spontanen Knochenneubil- dung im Osteotomiespalt (Kallo- bzw. Hemikallotaxis). Auch wenn dadurch eine Knochentransplantation vermieden werden kann, bevorzugen es die meisten Autoren, die gewünschte Länge und Form des Radius durch ein Aufspreizen der Osteotomie sofort intraoperativ einzustellen. Größe und Form des resultierenden Defektes hängen dabei von der Längendiskrepanz zwischen Radius und Ulna und der Verkippung des distalen Radiusfragmentes ab. Muss lediglich die dorsopalmare Neigung der Radiusgelenkfläche korrigiert werden, was häufiger bei fehlverheilten Smith- als bei fehlverheilten Colles-Frakturen der Fall ist, muss man bei der Osteotomie den Radius nicht vollständig durchtrennen. Bei Kippung der Radiusgelenkfläche nach dorsal kann die palmare, bei Kippung nach palmar die dorsale Kortikalis erhalten werden. Beim Aufspreizen resultiert ein dreieckiger Defekt. Stabilisiert man den Radius in diesen Fällen mit einem winkelstabilen Implantat, ist es ausreichend den Osteotomiespalt mit Spongiosa aufzufüllen. Beim Vergleich Spongiosa versus kortikospongiösem Knochenblock beobachteten Ring et al. [12] bei Verwendung von Spongiosa allein eher eine schnellere Durchbauung des Radius. Korrekturverluste traten bei Verwendung winkelstabiler Implantate in Kombination mit Spongiosa nicht auf.

Bei der typischen posttraumatischen Fehlstellung des distalen Radius mit Verkürzung des Radius, Abflachung der Ulnarinklination und Verkippung in der Sagittalebene muss man den Radius vollständig osteotomieren. Der entstehende Defekt ist sowohl in sagittaler als auch in frontaler Ebene trapezförmig und bedarf eines kortikospongiösen Knochenblockes.

Dieser wird meist vom Beckenkamm entnommen. Durman [4] berichtete 1935 über die Verwendung eines Knochenblockes aus dem Radius. Aus dem distalen Anteil des proximalen Radiusfragmentes wird ein monokortikaler Knochenblock longitudinal entnommen, um $90^{\circ}$ gedreht und in den Osteotomiespalt eingesetzt. Sennwald [14] veröffentlichte eine ähnliche Technik. Er schiebt den Knochenblock jedoch ohne ihn zu drehen nach distal (Griffelschachtelplastik). Wird gleichzeitig mit der Radiuskorrekturosteotomie ein resezierender Eingriff an der distalen Ulna vorgenommen, kann man die distale Ulna in den Osteotomiespalt interponieren [3]. Knochenersatzmaterial wurde vereinzelt - wie uns aus persönlichen Mitteilungen bekannt 


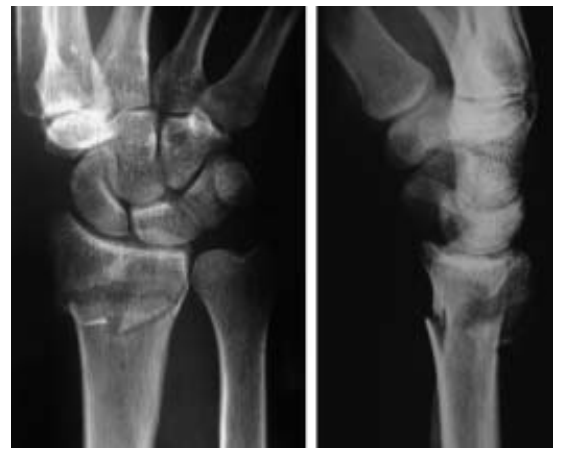

Abb. 2a Distale Radiusfraktur mit Dorsalkippung und Dorsalversatz des distalen Fragmentes.

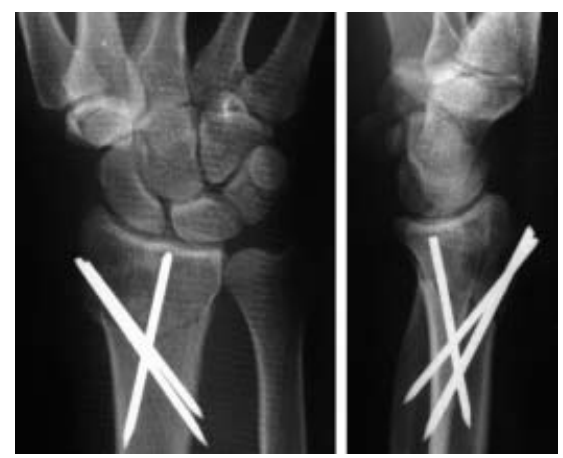

Abb.2b Operative Versorgung mit intrafokaler Drahtspickung.
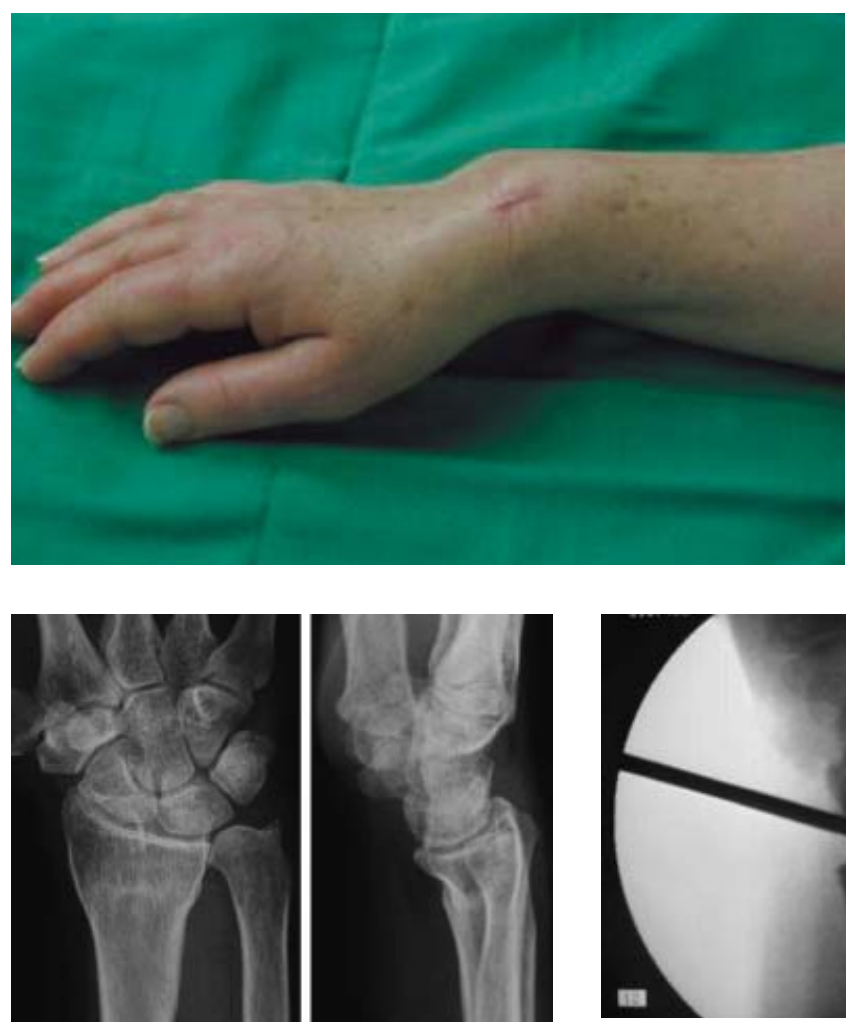

Abb.2d Im Röntgenbild findet sich eine vermehrte Palmarinklination der Radiusgelenkfläche bei leichter Radiusverkürzung.
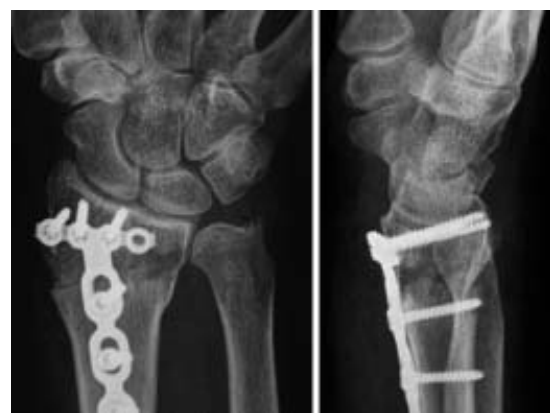

Abb.2f Frühpostoperativer Röntgenbefund.
Abb. 2c Ausheilungsergebnis nach Entfernung der Drähte: Klinisch zeigt sich die Handwurzel im Verhältnis zur Längsachse des Unterarmes nach palmar abgesagt. Supination des Unterarmes und Handgelenksextension waren deutlich eingeschränkt.

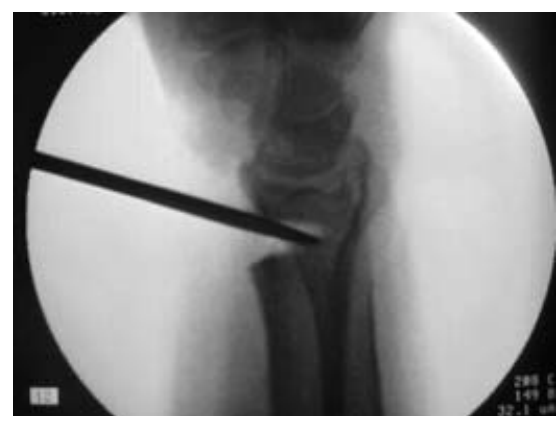

Abb.2e Radiuskorrekturosteotomie von palmar unter Erhalt der dorsalen Kortikalis.
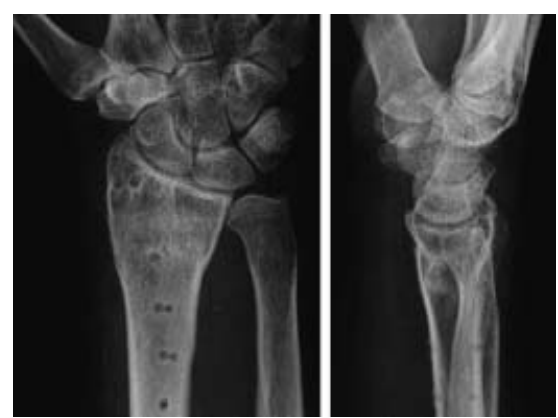

Abb. $\mathbf{2 g}$ Ausheilungsergebnis nach Entfernung des Osteosynthesemateriales ein Jahr nach Korrekturosteotomie.
- verwendet, um den Osteotomiespalt aufzufüllen. Publikationen hierüber sind bisher jedoch nicht erschienen.

\section{Ergebnisse}

Die Wiederherstellung des distalen Radius in Form und Länge führt zu einer Verbesserung der klinischen Situation: Die Schmerzen nehmen ab und die grobe Kraft nimmt zu, erreicht jedoch meist nicht das Niveau der unverletzten Gegenseite. Die Beweglichkeit des Handgelenkes sowie Unterarmdrehung zeigen sich postoperativ in allen Ebenen verbessert.

Eigene Untersuchungen zeigen, dass die Veränderungen von präoperativ nach postoperativ statistisch signifikant sind. Dies gilt sowohl für Korrekturosteotomien fehlverheilter Colles- als auch Smith-Frakturen [11]. So zeigte die Nachuntersuchung von 29 konsekutiven Patienten, bei denen wir zwischen Januar und Dezember 1995 bei einer fehlverheilten distalen Radiusfraktur vom Extensionstyp eine Korrekturosteotomie von radiopalmar in der von uns beschriebenen Technik durchgeführt hatten, 18 Monate (Minimum vier, Maximum 32 Monate) postoperativ alle radiologische $\mathrm{Pa}$ rameter hochsignifikant verbessert. Bei den klinischen Parametern fand sich eine hochsignifikante Steigerung der Handgelenksflexion, der Ulnarduktion und der groben Handkraft. Die Schmerzreduktion, erfasst auf der visuellen Analogskala von 0 bis 100 Punkten, war ebenfalls hochsignifikant (Tab.1).

Auch eine Nachuntersuchung von 20 Patienten, bei denen wir aufgrund einer fehlverheilten Flexionsfraktur des distalen Radius zwischen Mai 1992 und April 1999 eine Korrekturosteotomie vorgenommen hatten, zeigte bei einer durchschnittlichen Nachuntersuchungszeit von 18 Monaten (Minimum sechs, Maximum 36 Monate) alle radiologischen $\mathrm{Pa}$ rameter hochsignifikant im Vergleich $\mathrm{zu}$ präoperativ gebessert. Von den klinischen Parametern waren die Handgelenksextension signifikant, Ulnar-/Radialduktion, Supination und die Grobkraft hochsignifikant verbessert. Die Schmerzen zeigten sich postoperativ hochsignifikant gemindert. Handgelenksflexion und Unterarmpronation waren zwar klinisch gebessert, der Unterschied zu präoperativ war jedoch nicht statistisch signifikant (Tab.2). 
Tab. 1 Korrekturosteotomie fehlverheilter Colles-Frakturen $(n=29)$ : Verläufe der radiologischen und klinischen Parameter von prä- nach postoperativ (Mittelwerte \pm Standardabweichung; Signifikanztest: Wilcoxon-sign-rank-Test; Signifikanzlevel: $p \leq(0,05)$. Signifikante Unterschiede sind durch Fettdruck gekennzeichnet.

\begin{tabular}{|c|c|c|c|c|c|}
\hline \multirow{2}{*}{$\begin{array}{l}\text { Parameter } \\
\left.\text { Radiustilt ( }{ }^{\circ}\right)\end{array}$} & \multicolumn{2}{|c|}{ präoperativ } & \multicolumn{2}{|c|}{ postoperativ } & \multirow{2}{*}{$\begin{array}{l}\text { Signifikanz } \\
\mathbf{p}<\mathbf{0 . 0 0 1}\end{array}$} \\
\hline & 21 & $+I-10.7$ & -2.5 & $+I-7.1$ & \\
\hline Ulnarinklination $\left({ }^{\circ}\right)$ & 14 & $+I-7.0$ & 23 & $+I-5.9$ & $\mathbf{p}<0.001$ \\
\hline Ulnarvarianz (mm) & 4.5 & $+I-3.1$ & -0.5 & $+I-1.9$ & $\mathbf{p}<0.001$ \\
\hline Extension $\left({ }^{\circ}\right)$ & 42 & $+I-19.5$ & 48 & $+I-13.7$ & $p=0.028$ \\
\hline Flexion ( $\left.{ }^{\circ}\right)$ & 36 & $+I-20.3$ & 50 & $+I-17.6$ & $p=0.004$ \\
\hline Ulnarduktion ( ${ }^{\circ}$ ) & 15 & $+I-8.2$ & 21 & $+I-9.5$ & $p=0.01$ \\
\hline Radialduktion ( ${ }^{\circ}$ ) & 19 & $+I-12.9$ & 25 & $+I-11.1$ & $p=0.037$ \\
\hline Supination $\left({ }^{\circ}\right)$ & 73 & $+I-23.2$ & 78 & $+I-20.9$ & $p=0.029$ \\
\hline Pronation ( ${ }^{\circ}$ ) & 69 & $+1-25.2$ & 73 & $+1-22.2$ & $p=0.067$ \\
\hline Kraft (kpa) & 29 & $+I-18.2$ & 42 & $+I-22.5$ & $p=0.002$ \\
\hline Schmerzen (Punkte) & 70 & $+I-20$ & 27 & $+I-15$ & $\mathbf{p}<0.001$ \\
\hline
\end{tabular}

Tab. 2 Korrekturosteotomie fehlverheilter Smith-Frakturen $(n=20)$ : Verläufe der radiologischen und klinischen Parameter von prä- nach postoperativ (Mittelwerte \pm Standardabweichung; Signifikanztest: Wilcoxon-sign-rank-Test; Signifikanzlevel: $\mathrm{p} \leq 0,05)$. Signifikante Unterschiede sind durch Fettdruck gekennzeichnet.

\begin{tabular}{|c|c|c|c|}
\hline Parameter & präoperativ & postoperativ & Signifikanz \\
\hline Radiustilt ( ${ }^{\circ}$ ) & $-32+I-10.0$ & $+I-10.1$ & $\mathbf{p}<0.01$ \\
\hline Ulnarinklination $\left({ }^{\circ}\right)$ & $18+I-9.0$ & $29+I-7.1$ & $p<0.01$ \\
\hline Ulnarvarianz (mm) & $8+I-3.3$ & $1.0+I-2.5$ & $p<0.01$ \\
\hline Extension $\left({ }^{\circ}\right)$ & $42+I-13.7$ & $49+I-15.2$ & $p=0.039$ \\
\hline Flexion $\left({ }^{\circ}\right)$ & $47+1-22.0$ & $+\mid-21.0$ & $p=0.107$ \\
\hline Ulnarduktion ( ${ }^{\circ}$ ) & $21+I-12.0$ & $+I-9.5$ & $\mathbf{p}<0.01$ \\
\hline Radialduktion ( ${ }^{\circ}$ ) & $18+I-8.6$ & $+I-8.9$ & $p=0.01$ \\
\hline Supination ( $\left.{ }^{\circ}\right)$ & $47+I-28.1$ & $+I-21.0$ & $\mathbf{p}<0.01$ \\
\hline Pronation $\left({ }^{\circ}\right)$ & $73+\mid-19.9$ & $+\mid-14.4$ & $p=0.16$ \\
\hline Kraft (kpa) & $39+I-21.0$ & $+I-25.7$ & $p=0.02$ \\
\hline Schmerzen (Punkte) & $70+I-24$ & $+I-21$ & $\mathbf{p}<0.01$ \\
\hline
\end{tabular}

In einer retrospektiven Studie [11] untersuchten wir den Einfluss des radiologischen Ergebnisses auf das klinische Endresultat nach Korrekturosteotomie fehlverheilter distaler Radiusfrakturen und konnten zeigen, dass das klinische Endresultat umso besser ausfällt, je anatomischer der distale Radius in Form und Länge wiederhergestellt wird.

\section{Blick in die Zukunft}

So positiv die Ergebnisse nach Korrekturosteotomien fehlverheilter Radiusfrakturen auch sind, so darf man doch nicht vergessen, dass die meisten Radiuskorrekturosteotomien durch eine sorgfältigere Behandlung der primären Radiusfraktur vermeidbar wären. Die speziell für den distalen Radius neuentwickelten Implantate erlauben nahezu eine maßgeschneiderte Versorgung jeder Fraktur, auch bei älteren Menschen. Wir sollten diese Chancen nützen. Kommt es - trotzdem - zur Ausheilung einer distalen Radiusfraktur in Fehlstellung, muss man heute den betroffenen Patienten bei entsprechenden Beschwerden aufgrund der allgemein guten Ergebnisse eine Radiuskorrekturosteotomie anbieten. Ziel des Eingriffs muss die möglichst anatomiegerechte Wiederherstellung des Radius in Form und Länge sein, denn die klinischen Ergebnisse sind umso besser, je günstiger das radiologische Ergebnis ausfällt. Sowohl die Planung als auch die Durchführung der Radiuskorrekturosteotomie wird in naher Zukunft durch den Einsatz der Computertechnik erleichtert und verbessert werden.

\section{Literatur}

${ }^{1}$ Beyermann K, Prommersberger KJ, Krimmer $\mathrm{H}$ et al. Radio-scapho-lunate fusion as treatment of posttraumatic radiocarpal arthrosis. Eur J Trauma 2000; 26: 169-175

${ }^{2}$ Bowers WH. Distal radioulnar joint arthroplasty: The hemiresection-interposition technique. J Hand Surg 1985; 10A: 169-178

${ }^{3}$ Campbell WC. Malunited Colles' fractures. JAMA 1937; 109: 1105 - 1108

${ }^{4}$ Durman DC. An operation for correction of deformities of the wrist following fracture. J Bone Joint Surg 1935; 17: 1014-1016

${ }^{5}$ Fernandez DL. Correction of post-traumatic wrist deformity in adults by osteotomy, bone-grafting, and internal fixation. J Bone Joint Surg 1982; 64-A: 1164-1178

${ }^{6}$ Fernandez DL. Radial Osteotomy and Bowers Arthroplasty for malunited fractures of the distal end of the radius. J Bone Joint Surg 1988; 70-A: 1538 - 1551

${ }^{7}$ Jupiter JB, Ring D. A comparison of early and late reconstruction of malunited fractures of the distal end of the radius. J Bone Joint Surg 1996; 78-A: 739-748

${ }^{8}$ Lanz U. Kron W. Neue Technik zur Korrektur in Fehlstellung verheilter distaler Radiusfrakturen. Handchirurgie 1976; 8: 203-206

9 Prommersberger KJ, Lanz U. Die Korrekturosteotomie der fehlverheilten Radiusfraktur vom Extensionstyp. Operat Orthop Traumatol 1998; 10: 77-89

${ }^{10}$ Prommersberger KJ, Lanz U. Biomechanik der fehlverheilten distalen Radiusfraktur. Handchir Mikrochir Plast Chir 1999; 31: 221-226 

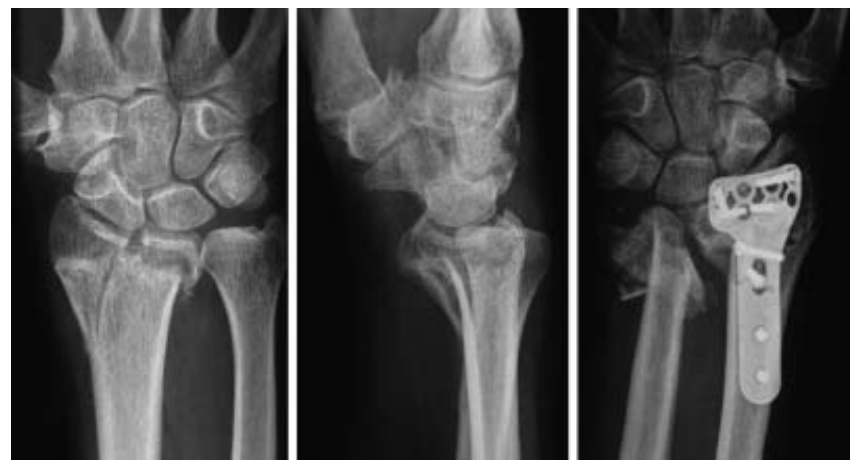

Abb. 3a Drei Monate nach Unfall zeigt das Röntgenbild des linken Handgelenkes in 2 Ebenen bei einem 65-jährigen Patienten eine mit einer Spaltbildung der Radiusgelenkfläche, einer Verkürzung des Radius im Verhältnis zur Ulna und einem Palmarversatz der Radiuskonsole ausgeheilte intraartikuläre Radiusfraktur. Die Gegenseite war zur OPPlanung bei trotz operativer Versorgung ebenfalls in Fehlstellung ausgeheilter distaler Radiusfraktur (noch zur Korrektur anstehend) nicht verwertbar.
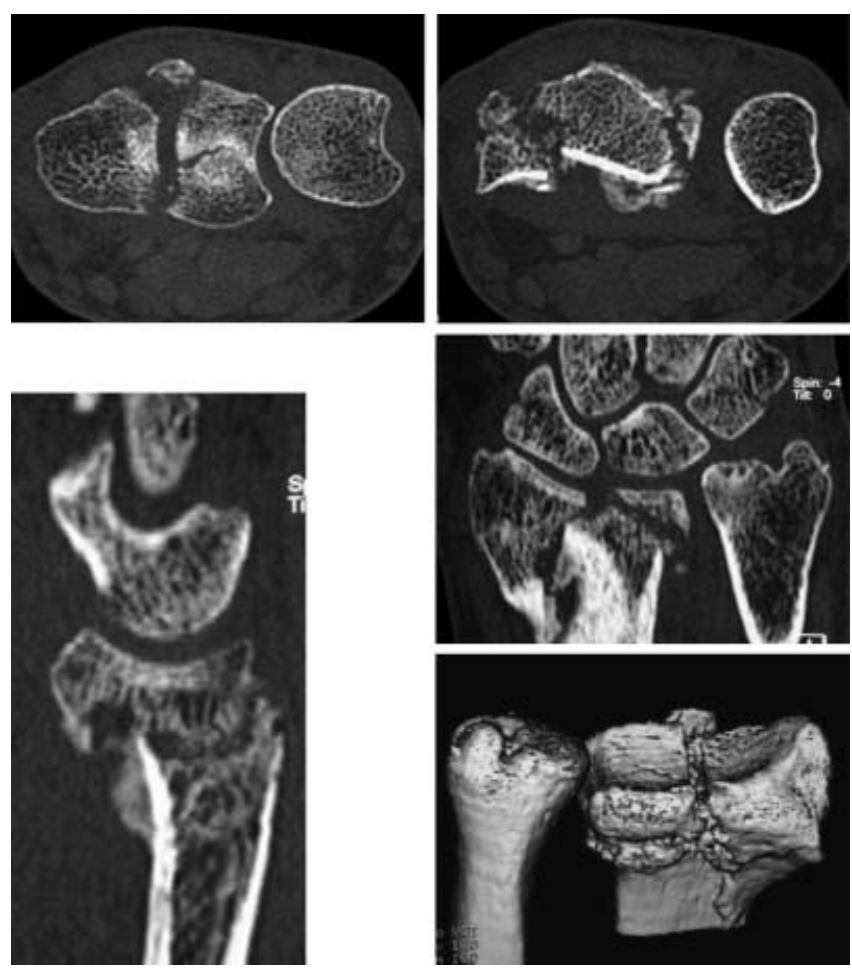

Abb.3b Präoperative Computertomographie zur OP-Planung, wobei insbesondere die 3D-Rekonstruktion hilfreich ist.

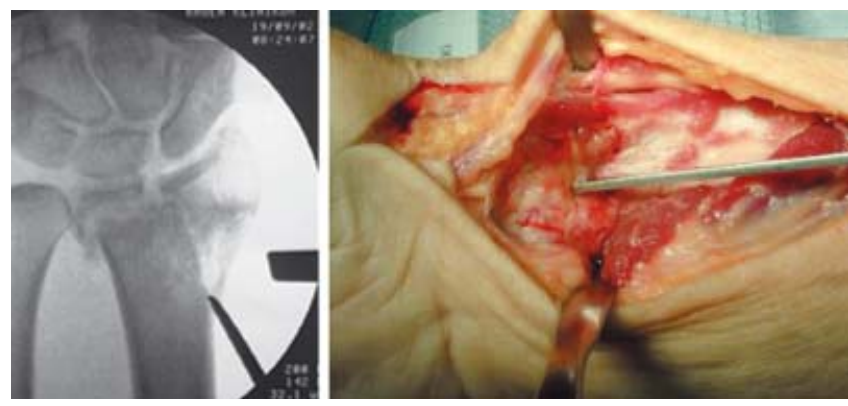

Abb.3c Osteotomie unter Durchleuchtungskontrolle.
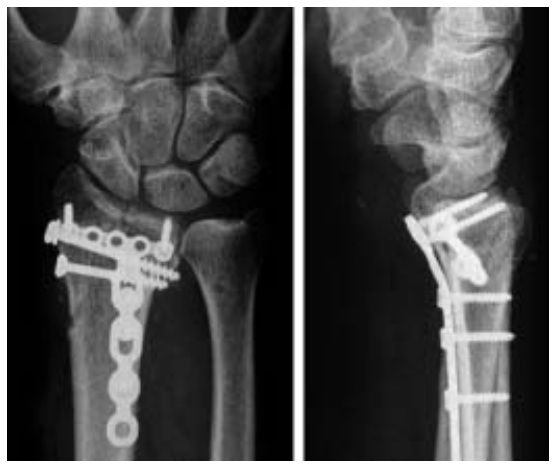

Abb.3d Postoperativer Nativ-Röntgenbefund.
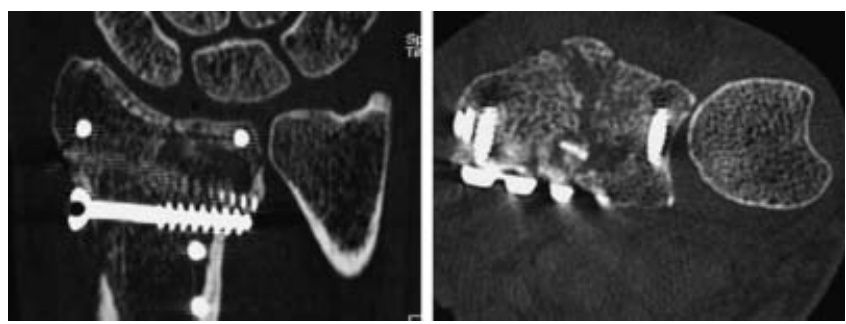

Abb.3e Postoperative Computertomographie: Die Spaltbildung der Radiusgelenkfläche und Palmarversatz der Radiuskonsole sind beseitigt und der Längenverlust ausgeglichen.
${ }^{11}$ Prommersberger KJ, Schoonhoven J van, Lanz U. Outcome after corrective osteotomy for malunited fractures of the distal end of the radius. J Hand Surg 2002; 27B: 55-60

${ }^{12}$ Ring D, Roberge C, Morgan T et al. Osteotomy for malunited fractures of the distal radius: A comparison of structural and nonstractural autogenous bone grafts. J Hand Surg 2002; 27A: 216-222
${ }^{13}$ Schoonhoven J van, Fernandez DL, Bowers WH et al. Salvage of failed resection arthroplasties of the distal radioulnar joint using a new ulnar head prosthesis. J Hand Surg 2000; 25A: 438-446

${ }^{14}$ Sennwald G, Fischer M. Correction du cal vicieux du radius distal par osteotomie de "glissement-avancement". Ann Chir Main 1993; 12: $124-129$

${ }^{15}$ Wright Th. Osteotomy for distal radius malunion. THUES 2000; 4: 222-235
Priv.-Doz. Dr. med. Karl-Josef Prommersberger

Oberarzt

Prof. Dr. med. Ulrich Lanz

Ärztlicher Direktor

Klinik für Handchirurgie

Salzburger Leite 1

D-97615 Bad Neustadt/Saale 